Photodynamic therapy

\section{Value based medicine}

\section{M Brown, G C Brown}

\author{
Let's get it right
}

$\mathrm{T}$ he article by Hopley and associates $\mathrm{n}$ this issue of the BJO (p 982) is a well performed study that utilises a form of healthcare economic analysis that is increasing in popularity throughout the healthcare literature. This instrument, cost utility analysis, has also been referred to by some as cost effectiveness analysis. A review of the healthcare literature using the key words "cost utility analysis" and "cost effectiveness analysis" reveals 386 hits for the years 1999 through 2003, 248 hits from years 1994 through 1998, and 109 hits from years 1989 through 1993. In year 1988 there were only five papers.

Cost utility analysis measures the value (improvement in quality of life and length of life) conferred by an intervention. It is the basic instrument that allows the creation of a value based medicine database to concomitantly improve quality of care at the same time as it maximises the efficiency of use of healthcare resources. ${ }^{2}$ Value based medicine is often a more accurate measure of the value conferred by healthcare interventions than evidence based medicine alone since value based medicine incorporates all of the quality of life parameters that evidence based outcomes-such as life expectancy, number of myocardial infarctions or strokes obviated, etc-typically ignore. Thus, the growing numbers of cost utility analyses bode well for both quality and efficiency in healthcare delivery.

There is a problem, however, in that very few cost utility analysis papers are comparable. Many studies use utility values to assess quality of life improvement, the costs are variable, but often comparable, and the discounting is relatively uniform. Then what is different?

The difference lies in the measurement of the utility values used to evaluate the improvement in quality of life. Utility values numerically quantify the quality of life associated with a health state. By convention, they range from 1.0 for perfect permanent health (or perfect permanent vision) to 0.0 for death. The higher the utility value, the better the quality of life associated with a health state. In ophthalmology, utility values are most highly correlated with the visual acuity in the better seeing eye.

The method of utility analysis, as well as the respondent source of utility values can make a dramatic difference in the quality of life measure for the same health state. Time trade-off utility analysis values differ considerably from standard gamble values and those obtained from patients who live with a disease frequently differ substantially from those obtained from physicians or the general community. ${ }^{23}$ The latter should not be contemplated any longer than it takes to read this paragraph. Utility value obtained from patients, alternatively known as patient preferences, should always be the "criterion" or gold standard. ${ }^{4}$

\section{We believe time trade-off utility analysis is the most reproducible and well understood by patients}

Can the most popular, current ophthalmic quality of life measures, the VF-14 and the National Eye Institute Visual Function Quotient-25 Question (NEI-VFQ-25) be used in cost utility analysis? Certainly not to date, and probably not in the future. Why? Because these ophthalmic measures of quality of life produce results that are not comparable with those obtained from quality of life instruments used in specialties outside ophthalmology. A major feature of a good quality of life instrument is that, in addition to being all encompassing of the variables that comprise quality of life, it should be applicable across all specialties. ${ }^{5}$ Utility analysis fits the criteria splendidly.

Until a standardised database of utility values is obtained from patients with diseases across all specialties in medicine, the great majority of cost utility analyses will not be comparable. Thus, the establishment of meaningful value based medicine standards will not be possible, and both quality of care and the efficiency of use of healthcare services will suffer.

Can it be done? Absolutely. The key is selecting a right preference based tool and sticking with it. After experimenting with multiple quality of life instruments, We believe time trade-off utility analysis is the most reproducible and well understood by patients. Once the standardised utility value database integrates the utility values associated with ophthalmic diseases with those associated with diseases across all of medicine, the value based sky is the limit.

Stay tuned.

Br J Ophthalmol 2004;88:979. doi: $10.1136 /$ bjo.2003.040030

Authors' affiliations

M M Brown, G C Brown, Center for ValueBased Medicine, 1107 Bethlehem Pike, Suite 210, Flourtown, PA 19031-1404, USA M M Brown, Department of Ophthalmology, University of Pennsylvania School of Medicine, Philadelphia, PA, USA

G C Brown, Retina Semice, Wills Eye

Hospital, Jefferson Medical College,

Philadelphia, PA, USA

Correspondence to: Melissa Brown, Center for Value-Based Medicine, 1107 Bethlehem Pike, Suite 210, Flourtown, PA 19031-1404, USA; lissa1011@aol.com

Accepted for publication 16 January 2004

\section{REFERENCES}

1 Gold MR, Patrick DL, Torrance GW, et al. Identifying and valuing outcomes. In: Gold MR, Siegel JE, Russell LB, Weinstein MC, eds. Costeffectiveness in health and medicine. New York Oxford University Press, 1996:82-134.

2 Brown MM, Brown GC, Sharma S, et al. Health care economic analyses and value-based medicine. Surv Ophthalmol 2003;48:204-23.

3 Brown GC, Brown MM, Sharma S. Difference between ophthalmologist and patient perceptions of quality-of-life associated with age-related macular degeneration. Can J Ophthalmol 2000;35:27-32

4 Brown MM, Brown GC, Sharma S. Value-based medicine. AMA Press (in press). 